\title{
Hepatic angiosarcoma with an associated focal nodular hyperplasia-like nodule
}

\author{
Sho Ogata ${ }^{1 *}$, Takuya Horio ${ }^{2}$, Susumu Tominaga ${ }^{1}$ and Yoshiaki Sugiura ${ }^{2,3}$ \\ ${ }^{1}$ Department of Pathology and Laboratory Medicine, National Defense Medical College, Tokorozawa, Japan \\ ${ }^{2}$ Departments of Surgery, National Defense Medical College, Tokorozawa, Japan \\ ${ }^{3}$ Department of Surgery and Gastroenterology, International University of Health and Welfare Mita Hospital, Tokyo, Japan
}

\section{Introduction}

Hepatic angiosarcoma (AS) is a rare malignant tumor exhibiting proliferation of the malignant endothelial cells and the formation of irregular vascular channels. We present a case in which a hepatic AS was accompanied by a nearby focal nodular hyperplasia (FNH)-like nodule within a fibrotic alcoholic liver. A hypervascular hepatic mass was found and a biopsy specimen taken from the mass was suspected to be a hyperplastic nodule. However, the follow-up ultrasonography, which was performed a half-year later, detected a large, hypervascular tumor at that site, and histology revealed an AS. In the resected liver specimen, which displayed moderate fibrosis, a small mass was found close to the AS. That small mass was composed of hyperplastic hepatocytes with fibrotic septa and abnormal vessels. No normal portal areas were evident, and so it was considered to be an FNH-like nodule. Immunohistochemically, sinusoids were highlighted by CD34 and angiopoietin-1. Abnormal vessels were also observed outside this FNHlike nodule. Possibly, an abnormal vasculature may have provided these lesions with a common pathogenic background, and secreted growth factors might have synergic effects on the growth of the lesions.

Hepatic angiosarcoma (AS) is a rare malignant mesenchymal tumor with an extremely poor prognosis [1,2]. It is characterized histologically by the presence of malignant cells forming anastomosed, irregular vascular channels $[1,2]$. Focal nodular hyperplasia $(\mathrm{FNH})$, on the other hand, is a benign hepatic lesion displaying hepatocytic hyperplasia with a fibrous center containing an abnormal vasculature [3-5]. FNH and FNH-like nodule, the latter a histologic mimic of FNH within a fibrotic liver [6], have been considered to be caused by an abnormal intrahepatic vascular supply [4,7]. However, to our knowledge there has been no report concerning a hepatic AS accompanied by FNH or FNHlike nodule. Here, we present a Japanese case with a ruptured hepatic AS accompanied by an FNH-like nodule within a fibrotic alcoholic liver, and we describe the lesions' features, and discuss their association.

\section{Case report}

A 67-year-old Japanese man with alcoholic liver disease had been followed at the National Defense Medical College Hospital (Saitama, Japan), after his first referral because of elevations in liver enzyme values found in a medical check-up. He had been a habitual drinker (intake, more than $65 \mathrm{~g}$ of alcohol/day for 40 years). He had a previous history of operations for hemorrhoids and for intervertebral disc herniation, although he had not been exposed to Throtorast, arsenic, or vinyl chloride compound. His laboratory data revealed no hepatitis $\mathrm{B}$ or $\mathrm{C}$ viral antigen or antibody. After five-year-follow-up, abdominal ultrasonography revealed a roughly one $\mathrm{cm}$-sized nodule within the lateral liver segment, and contrast computed tomography revealed early enhancement of the nodule. Specimens from repeatedly performed needle biopsies exhibited hepatocytic hyperplasia with mild structural atypia and sinusoidal dilatation, leading to suspicion of a hyperplastic nodule. Focal fatty change with ballooning degeneration was observed within the background liver parenchyma. However, the follow-up imaging studies performed a half-year later found, unexpectedly, a hypervascular mass sized $70 \mathrm{~mm}$ at the almost same site (Figure 1A). A hepatocellular carcinoma was suspected because of the results of the previous liver biopsy, although serum tumor markers (alpha fetoprotein and protein-induced by vitamin $\mathrm{K}$ absence or antagonist-II) were within their normal ranges. However, histologic examination of a needle biopsy specimen taken from the tumor led us to suspect an AS. Lateral liver segmentectomy was scheduled.

After receiving a preoperative enema on the morning of the operation day, he complained of feeling vaguely ill. During induction of anesthesia in the operation room, his systolic blood pressure suddenly fell to the $50 \mathrm{mmHg}$ level. An urgent laparotomy revealed that the tumor had ruptured, causing a massive intraperitoneal hemorrhage. The tumor was resected by lateral segmentectomy, and he recovered from the shock state. His post-operative course was uneventful. He underwent transcatheter arterial chemoembolization (lipiodol + adriamycin + mitomycin) and radiofrequency ablation, and was given systemic docetaxel and interleukin-2 because he was found to have a metastatic deposit within the right liver lobe. Despite this, the recurrent tumor grew and he was accompanied by chronic disseminated intravascular coagulation. Finally, he died following rupture of a metastatic lung tumor 11 months after the operation. Unfortunately, an autopsy was not performed.

Within the segmentectomy specimen, we found two distinct tumorous lesions (Figure 1B,C). One was a well-demarcated, $7 \mathrm{~cm}$ sized, hemorrhagic tumor located in the subcapsular region and rupturing into the abdominal cavity, where it was responsible for the massive intraperitoneal hemorrhage. Histology revealed that this

${ }^{\star}$ Correspondence to: Dr. Sho Ogata, Department of Pathology and Laboratory Medicine, National Defense Medical College, 3-2 Namiki, Tokorozawa Saitama, 359-8513 Japan, Tel: +81-4-2995-1505; Fax: +81-4-2996-5192; E-mail: sogata@ndmc.ac.jp

Key words: alcoholic liver disease; angiosarcoma; focal nodular hyperplasia

Received: April 06, 2018; Accepted: April 30, 2018; Published: May 5, 2018 


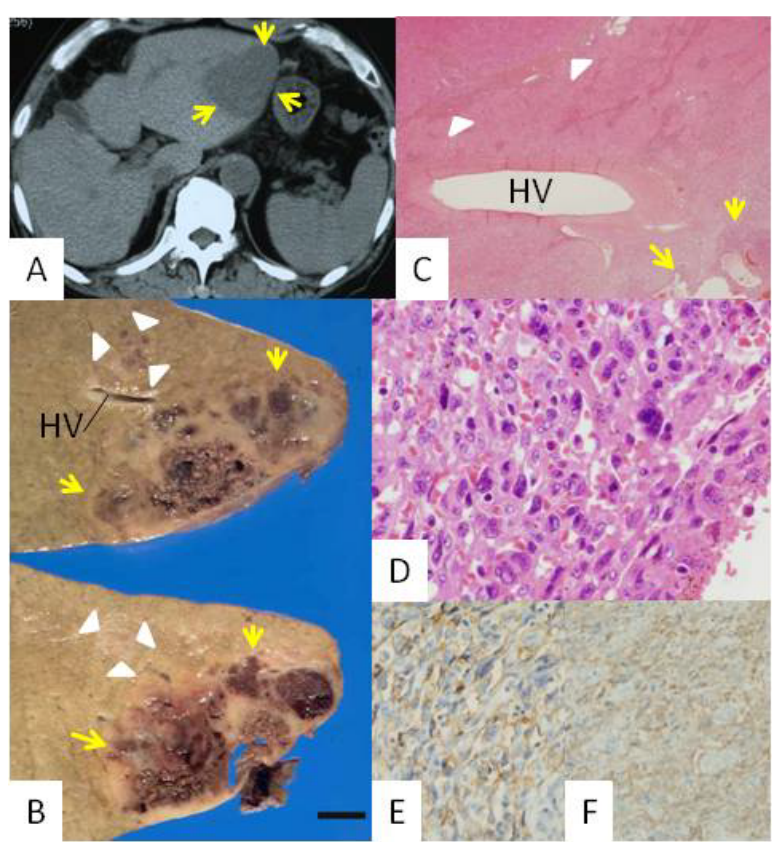

Figure 1. Features of the hepatic angiosarcoma (AS)

A. Abdominal computed tomography revealed a low-density tumor (indicated by yellow arrows) within the lateral liver segment

B, C. Within the resected (B) and histologic (C) specimens, in addition the rupturing main tumor, a small nodule (white arrowheads) was found in the vicinity of the AS. HV in B and $\mathrm{C}$ indicates a hepatic vein, and the black bar in B indicates $1 \mathrm{~cm}$

D. Histology revealed vasofomation by anaplastic tumor cells, consistent with AS

E, F. AS cells were immunoreactive for CD34 (E) and angiopoietin-1 (F) (C, D, hematoxylin-eosin; E, F, diaminobenzidine)

tumor consisted of anaplastic tumor cells that had proliferated to form complex vascular channels (Figure 1D), sometimes interposed by fibrous tissue. At the periphery, the tumor cells extended in a sinusoidal fashion. The tumor cells were immunoreactive for anti-CD34 (Figure 1E) and anti-factor VIII antibodies. These findings were compatible with it being an AS.

The second, gray, lesion, $1.4 \times 1.3 \times 1.0 \mathrm{~cm}$-sized, was located in the close proximity to the above AS (Figure 1B,C). Histology revealed that the second lesion was a hyperplastic hepatocytic lesion interposed irregularly by fibrous septa and partially surrounded by a fibrous capsule (Figure 2A). The fibrous septa contained thick-walled vessels (Figure 2B, C) and cholangioles. Neither apparent portal veins nor any bile ducts were observed. Cellular atypia among the involved hepatocytes was indistinct, and its cellularity was considered to represent a slight increase. Capillarization of the sinusoids within the lesion was confirmed immunologically by anti-CD34 antibody (Figure 2D). Moreover, both the lesion and the AS were immunoreactive for angiopoietin-1 (Ang1 ), an angiogenic growth factor (Figures $1 \mathrm{~F}$ and $2 \mathrm{E}$ ). The lesion's morphology mimicked that of FNH, and so it was diagnosed as an FNH-like nodule because the background liver parenchyma displayed a moderate degree of fibrosis. The background liver parenchyma was not associated with fatty degeneration or ballooning degeneration although these findings had been observed within previous biopsy specimens. Thick-walled vessels were also observed outside this FNH-like nodule, (Figure 2F).

\section{Discussion}

Although a few cases of hepatic AS within a fibrotic alcoholic liver have been reported, the pathogenicity of AS in alcoholic liver fibrosis remains unclear [8-10]. We present here AS accompanied by an FNHlike nodule. We found the AS during the period of follow-up for the FNH-like nodule, and it had evidently grown rapidly to $70 \mathrm{~mm}$ in size during the preceding a half-year. In the segmentectomy specimens, AS and the FNH-like nodule were located in intimate association, and abnormal blood vessels were also found, even outside the FNH-like nodule. In general, hypervascular hyperplastic nodules including FNHlike nodules are known to occur in alcoholic liver fibrosis, and FNH and FNH-like nodules have been considered to be localized lesions resulting from reactions to an abnormal circulatory change $[3,4,6,7,11,12]$. In fact, FNH is frequently associated with hepatic hemangioma, a benign counterpart of AS $[2,13]$. On the other hand, there is no published report of an association between abnormal vasculature and the pathogenesis of AS. However, in some cases AS has been reported to be in association with hepatic nodular regenerative hyperplasia, which exhibits diffuse hepatocytic hyperplasia due to abnormal circulatory disturbance throughout the whole liver $[2,4,7]$. On the basis of the above findings, we suspected that in the present case the AS and the FNH-like nodule may each have induced a positive response in the other, and that the increased blood-burden to the endothelium might have been partly responsible for the rapid development of the AS.

In the present case, sinusoidal capillarization was distributed throughout the FNH-like lesion, and both this capillarization and the AS cells expressed Ang-1. Recently, FNH was found to overexpress Ang-1, and transgenic mice with overexpression of an Ang-1 in their hepatocytes exhibited abnormal hepatic vasculature and hepatocytic nodular proliferation [14,15]. Further, AS has been reported to overexpress the receptor for Ang-1 [16,17]. The expressions of angiogenic growth factors in the present case might suggest a pathogenic

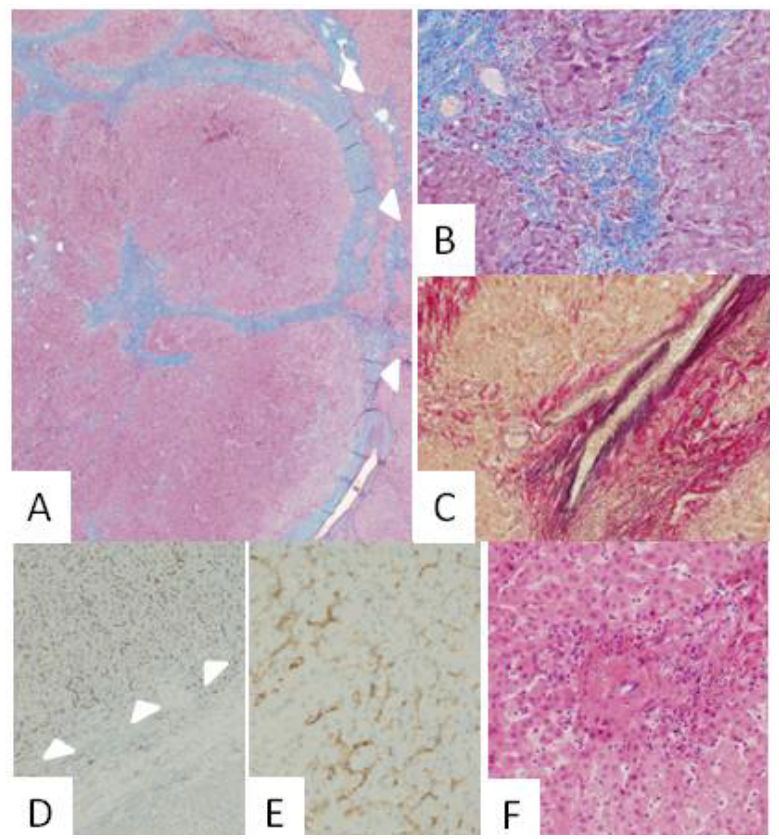

Figure 2. Histologic features of the focal nodular hyperplasia-like nodule

A. The nodule (indicated by white arrows) had an incomplete fibrous capsule and septa B, C. Within the nodule, portal areas were not evident, and the fibrous septa contained arterial vessels

D. Immunohistochemical CD34 staining revealed sinusoidal staining within the nodule (white arrowheads). Note also the negative reaction of the background liver (lower field in the figure)

E. Angiopoietin-1 was expressed in the nodule

F. A thick-walled vessel was also observed outside the nodule. (A, B, Masson trichrome; C, elastica van Gieson; D, E, diaminobenzidine; F, hematoxylin-eosin) 
linkage between the lesion and the AS: possibly, Ang-1 secreted from the lesion and from the AS itself may have stimulated the growth of the AS. Our findings are consisted with this notion.

In conclusion, we have presented a case with a hepatic AS accompanied by a nearby FNH-like nodule. Although this is a rare association, it may result from the presence of abnormal vasculature and a local secretion of growth factors.

\section{References}

1. Miettinen M, Fletcher CDM, Kindblom LG, Zimmermann A, Tsui (2010) WMS: Mesenchymal tumours of the liver. In: Bosman FT, Carneiro F, Hruban RH, Theise ND eds. WHO classification of tumours of the digestive system, 4th edn Lyon: IARC Press; 241-250.

2. Ishak KG, Goodman ZD, Stocker JT (2001) Tumors of the liver and intrahepatic bile ducts. Rosai J ed. Atlas of tumor pathology. 3rd series Washington, DC: Armed Forces Institutes of Pathology.

3. Bioulac-Sage P, Balabaud C, Wanless I (2010) Focal nodular hyperplasia and hepatocellular adenoma. In: Bosman FT, Carneiro F, Hruban RH, Theise ND eds. WHO classification of tumours of the digestive system, 4th edn Lyon: IARC Press, 198-204.

4. Kondo F (2001) Benign nodular hepatocellular lesions caused by abnormal hepatic circulation: etiological analysis and introduction of a new concept. $J$ Gastroenterol Hepatol 16: 1319-1328. [Crossref]

5. International Working Party (1995) Terminology of nodular hepatocellular lesions. Hepatology 22: 983-993. [Crossref]

6. Nakashima O, Kurogi M, Yamaguchi R, Miyaaki H, Fujimoto M, et al. (2004) Unique hypervascular nodules in alcoholic liver cirrhosis: identical to focal nodular hyperplasia-like nodule? J Hepatol 41: 992-998. [Crossref]

7. Kondo F, Kondo K, Nagao T, Sato T, Tomizawa M, et al. (1998) Etiological analysis of focal nodular hyperplasia of the liver: with emphasis on similar abnormal vasculatures to nodular regenerative hyperplasia and idiopathic portal hypertension. Pathol Res Pract 194: 487-495. [Crossref]

8. Saleh HA, Tao LC (1998) Hepatic angiosarcoma: aspiration biopsy cytology and immunocytochemical contribution. Diagn Cytopathol 18: 208-211. [Crossref]

9. Kahraman A, Miller M, Baba H, Gerken G, Hilgard P (2006) Angiosarcoma of the liver as a rare cause of rapidly progressive liver failure. Med Klin (Munich) 101: 746-750 [Crossref]

10. Murawaki Y, Kono M, Muira M, Sugihara T, Tanimura T, et al. (2011) A case of hepatic angiosarcoma detected as a small lesion. Nihon Shokakibyo Gakkai Zasshi 108: 12521262. [Crossref]

11. Gludd C, Christofferson P, Eriksen J, Wantzin P, Knudsen BB (1987) Influence of ethanol on development of hyperplastic nodules in alcoholic men with micronodular cirrhosis. Gastroenterology 93: 256-260. [Crossref]

12. Moon JH, Ahn CM, Chung HS, Ahn SH, Park YN (2006) A case of hypervascular hyperplastic nodules in a patient with alcoholic liver cirrhosis. Yonsei Med J 47: 881886. [Crossref]

13. Vilgrain V, Uzan F, Brancatelli G, Federle MP, Zappa M, et al. (2003) Prevalence of hepatic hemangioma in patients with focal nodular hyperplasia: MR imaging analysis. Radiology 229: 75-79.

14. Gouw A, Zeng W, Buislool M, Platteel I, van den Heuvel MC, et al. (2010) Molecular characterization of the vascular features of focal nodular hyperplasia and hepatocellular adenoma: a role of angiopoietin-1. Hepatology 52: 540-549. [Crossref]

15. Ward NL, Haninec AL, Slyke PV, Sled JG, Sturk C, et al. (2004) Angiopoietin-1 causes reversible degradation of the portal microcirculation in mice. Implication for treatment of liver disease. Am J Pathol 165: 889-899. [Crossref]

16. Amo Y, Masuzawa M, Hamada Y, Katsuoka K (2004) Observations on angiopoietin 2 in patients with angiosarcoma. Br J Dermatol 150: 1028-1029. [Crossref]

17. Brown LF, Dezube BJ, Tognazzi K, Dvorak HF, Yancopoulos GD (2000) Expression of Tie1, Tie2, and angiopoietins 1, 2, and 4 in Kaposi's sarcoma and cutaneous angiosarcoma. Am J Pathol 156: 2179-2183. [Crossref]

Copyright: (C2018 Ogata S. This is an open-access article distributed under the terms of the Creative Commons Attribution License, which permits unrestricted use, distribution, and reproduction in any medium, provided the original author and source are credited. 